\title{
When Moons Create Aurora: The Satellite Footprints on Giant Planets
}

\author{
B. Bonfond \\ Laboratoire de Physique Atmosphérique et Planétaire, Université de Liège, Liège, Belgium
}

\begin{abstract}
Satellite footprints are localized auroral emissions in the upper atmosphere of Jupiter (Saturn) near the magnetic field lines linking to satellites, Io, Europa, and Ganymede (Enceladus). They are the auroral signatures of the strong electromagnetic interactions taking place between these moons and the intensely magnetized, rapidly rotating planets they orbit. The Io and Europa spots have been shown to be followed by an extended tail. This might also be the case for the Ganymede and Enceladus emissions, although not yet unambiguously observed. Moreover, the main Io spot is accompanied by secondary spots attributed either to reflections of the plasma waves generated at Io on the Io plasma torus boundary or to electrons accelerated in one hemisphere but precipitating in the opposite one. While the horizontal extent of the spots gives a hint of the size of the interaction region in the equatorial plane, the vertical profile of the footprints provides clues to the energy distribution of the precipitating electrons. Moreover, the location of the footprints can be used as constraints for magnetic field models. Finally, the brightness of the footprints is a valuable diagnostic of the interaction mechanism and has been observed to vary at different time scales, each one tentatively associated with a different process.
\end{abstract}

\section{INTRODUCTION}

Jupiter is a source of intense radio emissions, the observation (from Earth) of which was found to be highly correlated with the orbital position of the satellite Io [Bigg, 1964]. This led Goldreich and Lynden-Bell [1969] to propose an electromagnetic interaction between Jupiter and Io that ultimately gives rise to radio emissions from the foot of the Io flux tube in Jupiter's upper atmosphere. Io's volcanism supplies a neutral torus that ultimately feeds a dense plasma that migrates outward, forming an equatorial plasma sheet. Jupiter's magnetic dipole is tilted some $9.6^{\circ}$ from its rotation axis. Cold iogenic plasma corotating with the magnetic field is confined by centrifugal forces to the centrifugal equator, a plane about two thirds of the way between the rotation and

Auroral Phenomenology and Magnetospheric Processes: Earth and Other Planets

Geophysical Monograph Series 197

(C) 2012. American Geophysical Union. All Rights Reserved.

10.1029/2011GM001169 magnetic equators [Gledhill, 1967]. Consequently, as the plasma sheet rotates with Jupiter, it passes over orbiting satellites twice per rotation where the centrifugal and rotation planes intersect. Viewed in a frame of reference at rest with the plasma sheet, the satellites move up and down in harmonic motion about the centrifugal equator, as a function of their System III longitude.

At Saturn, Enceladus's cryovolcanism is also a major plasma source in the Kronian magnetosphere [Pontius and Hill, 2006]. Enceladus is also embedded in a plasma torus, but it always remains constantly 0.04 Saturn radius below its center because Saturn's rotation axis and its magnetic dipole are nearly coaligned, but the magnetic equator is offset relative to the rotational one.

In brief, the basic scenario to generate auroral footprints is the following one. The satellites constitute obstacles to the corotating plasma flow, which overtakes their much slower prograde orbital motion. This perturbation propagates along the magnetic field lines as Alfvén waves, and the locus of the perturbed points is the Alfvén wing. On their way to the planet, the waves cause the acceleration of 
electrons, which finally precipitate into the planetary atmosphere and trigger aurora [Hess and Delamere, this volume]. The auroral spots may appear downstream of the foot of an undisturbed magnetic field line traced from the satellite to the top of Jupiter's atmosphere, due to complexities of the interaction. For example, in the ideal Alfvén wing scenario [Neubauer, 1980; Goertz, 1980], the wing is inclined with respect to the background magnetic field because of the combination of the flux tube motion relative to the satellite and the finite propagation speed of the Alfvénic disturbance. The downstream shift of the most prominent ("main") spot along the footpath, is called the "lead angle." However, its measurement is delicate because of the limited accuracy of the magnetic field models [Bonfond et al., 2009]. This terse summary does not do justice to the complexity and to the diversity of the phenomena taking place close to the satellites (see reviews by Kivelson et al. [2004], Saur et al. [2004], and Jia et al. [2009]).

The first satellite footprint to be detected was the Io footprint, as its $\mathrm{H}_{3}^{+}$emissions were identified in the infrared domain by Connerney et al. [1993]. This finding was confirmed in the FUV (120-170 nm) by Hubble Space Telescope (HST) observations [Prangé et al., 1996; Clarke et al., 1996]. No other footprint has been observed in the IR domain since. Moreover, the Io footprint is also the only one that has been detected in visible wavelengths (by the Galileo and New Horizons probes) [Vasavada et al., 1999; Gladstone et al., 2007]. The Europa and Ganymede footprints were simultaneously discovered in 2002 with the HST in the UV range [Clarke et al., 2002]. The Enceladus footprint was discovered in 2011 with the EUV and FUV channels of the Ultraviolet Imaging Spectrograph (UVIS) instrument aboard the Cassini probe [Pryor et al., 2011; Gurnett and Pryor, this volume] (Figure 1).

\section{MORPHOLOGY OF THE SATELLITE FOOTPRINTS}

Up to now, the Europa, Ganymede, and Enceladus footprints have been seen as single spots in each hemisphere. However, based on images from NASA's Infrared Telescope Facility (IRTF), Connerney and Satoh [2000] reported an Io footprint made of five equally spaced spots. Moreover, on UV images, up to three Io footprint spots can be distinguished along with an extended downstream tail (Figure 2) [Clarke et al., 2002; Gérard et al., 2006; Bonfond et al., 2008]. For each satellite, the locus of these features forms a closed contour in a System III reference frame, which is called the satellite footpath. The Europa spot is also sometimes followed by a short tail [Grodent et al., 2006]. Furthermore, these Io spots appear to move with respect to each other as Io moves up and down inside the plasma torus. The multiplicity of these spots first suggested that they were due to reflections of the Alfvén waves on the density gradient at the plasma torus boundary as illustrated by the blue trajectories in Figure 2 [Neubauer, 1980; Gurnett and Goertz, 1981]. The main Alfvén wing (MAW) spot would be located at the feet of a direct Alfvén wing, while subsequent ones would be related to reflected Alfvén wings (RAW). Another explanation could be that the multiple spots are not the direct counterpart of RAW, but an interference pattern due to the multiple reflections [Jacobsen et al., 2007]. The finding of a faint spot emerging upstream of the brightest one in one hemisphere while only downstream spots are seen in the opposite hemisphere challenged these hypotheses. Bonfond et al. [2008] suggested that, additionally to the MAW spot and to the RAW spot, one of the spots, called the transhemispheric electron beam (TEB) spot, is caused by electrons accelerated away from the planet in one hemisphere and precipitating in the opposite hemisphere (Figure 2). This scenario does not only account both qualitatively
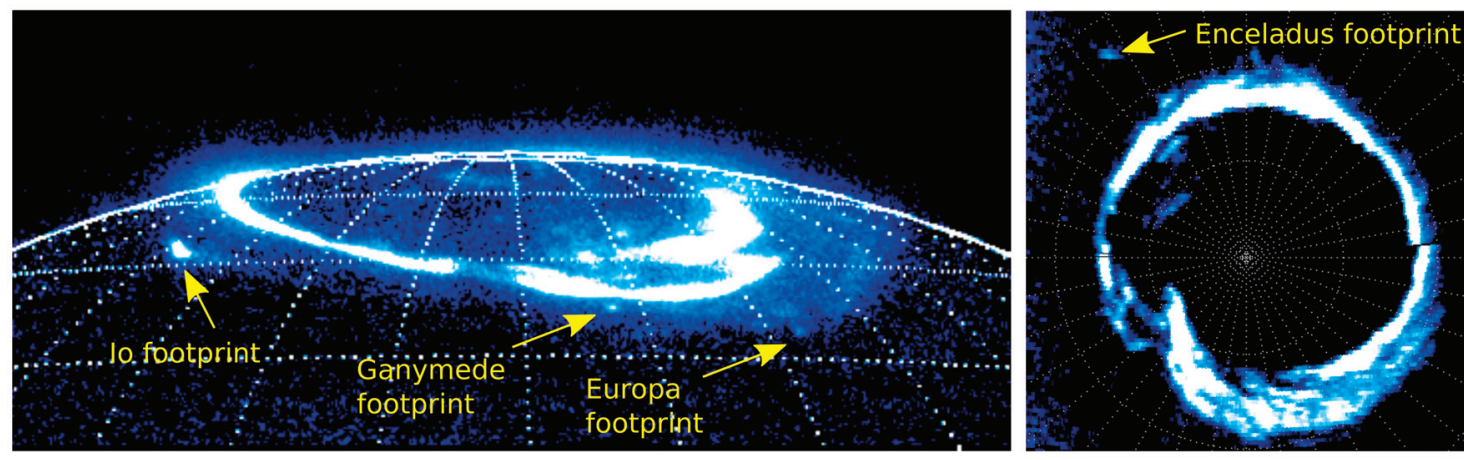

Figure 1. (left) Image of the northern FUV aurora of Jupiter acquired with the Hubble Space Telescope's ACS camera on 7 February 2006. The Io, Europa, and Ganymede footprints are simultaneously visible. (right) Polar projection of Saturn's FUV aurora as observed by Cassini's UVIS instrument on 26 August 2008 (D. Grodent and the Cassini/UVIS team). The Enceladus footprint is visible more equatorward than any other auroral emission. 

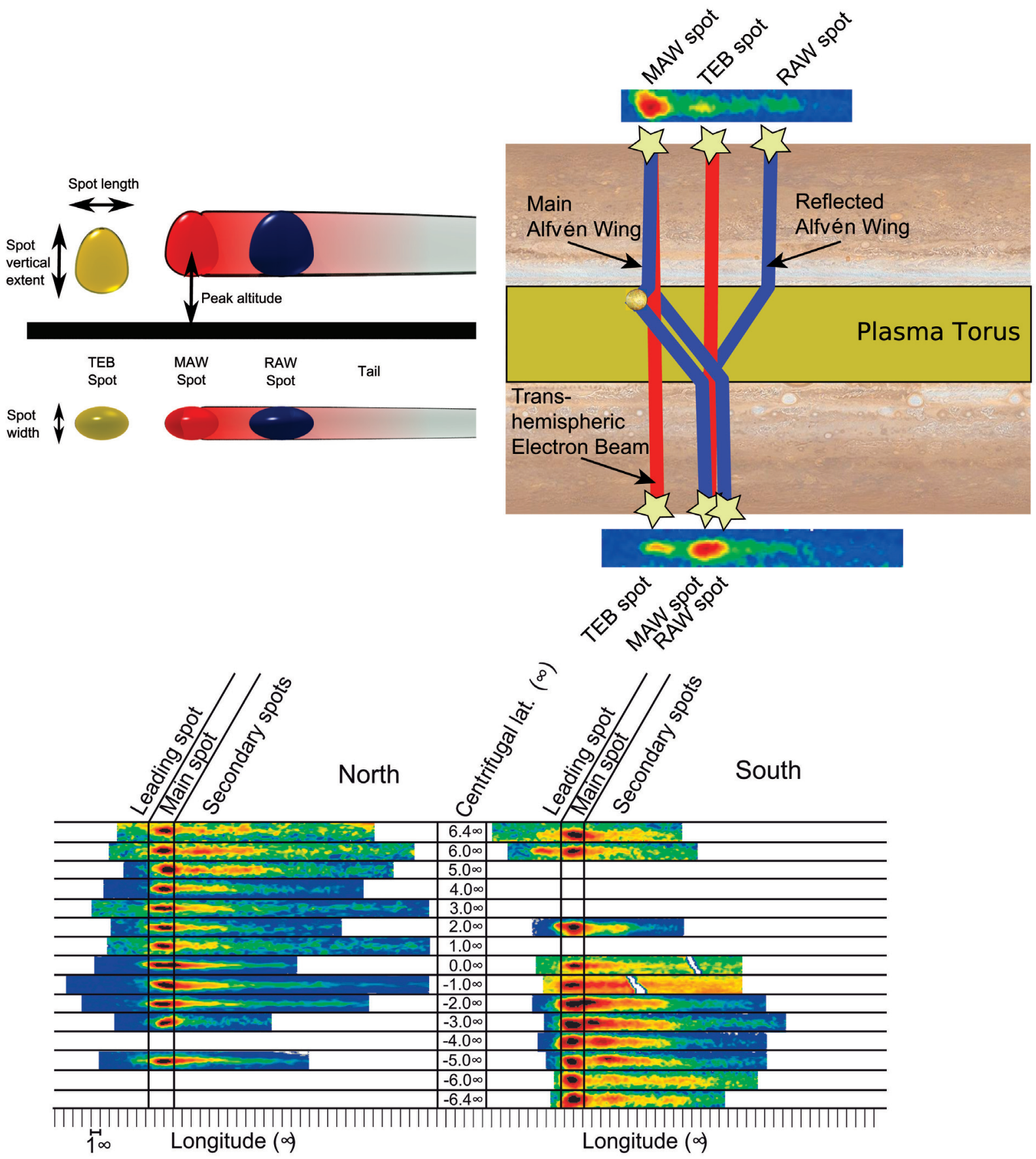

Figure 2. (top left) A sketch of the Io footprint as seen from the side (top diagram) and from above (bottom diagram). The Io footprint is considered to be formed by three spots and an extended downstream tail. (top right) Geometry of the Alfvén wave propagation and their reflection against the inner boundary of the torus. The direct Alfvén wing generates the main Alfvén wing spots, while the reflected wings create the reflected Alfvén wing spots. In contrast to the Alfvén waves, the electron beams are not affected by the high torus density, which enables them to propagate rapidly from one hemisphere to the other, generating the transhemispheric electron beam spots. (bottom) Io footprint morphology as a function of the centrifugal latitude of Io in the torus. The color table of each stripe is scaled individually for a clearer illustration of the morphology. The longitudes are not measured on the planet but mapped to the equatorial plane along the magnetic field lines according to the VIP4 model for an easier comparison of both hemispheres. Adapted from Bonfond et al. [2008] and Bonfond [2010]. 
and quantitatively for the evolution of the interspot distances, but it also explains the occurrence of bidirectional electron beams close to Io [Williams and Thorne, 2003]. Indeed, Jacobsen et al. [2010] computed the magnetic field line bending due to the Io-magnetosphere interaction with a nonlinear 3-D MHD model and showed that the scenario of the TEBs would account for the location of the electron beams as observed when Galileo flew by Io. Saur et al. [2002] and Dols et al. [2008] discovered that these energetic electron beams were an additional ionization source for Io's stagnant plasma wake. Moreover, Wilkinson [1998] argued that the periodicity of Io-related decametric radio emissions could arise from a similar mechanism. Several models [Hill and Vasyliūnas, 2002; Delamere et al., 2003; Ergun et al., 2009] describe the Io footprint tail as caused by a steady state process due to the progressive reacceleration of the plasma downstream of Io. On the other hand, MHD simulations indicate that it might actually be the result of multiple reflections of the Alfvén waves [Jacobsen et al., 2007].

The presence of electron beams and their probable relationship with the auroral footprint has also been highlighted at Enceladus [Pryor et al., 2011]. Unlike Jupiter, the Kronian magnetic field dipole axis is perpendicular to the satellites' orbital plane within measurement accuracy, and Enceladus remains $\sim 10$ Enceladus radii southward from the torus center. Consequently, the distance between the MAW spot and the TEB spot should remain small and constant.

\section{LOCATION OF THE SATELLITE FOOTPRINTS}

Mapping a position in the magnetosphere to its ionospheric foot can be challenging if the field is not well known along any portion of its path, which routinely occurs in the outer magnetosphere of the planets, linking to polar magnetic latitudes. However, when moons generate auroral footprints, the direct connections between the two phenomena provide indisputable landmarks in the magnetosphere. For example, Radioti et al. [2009] used tabulated Ganymede footprint positions to discuss the mapping of the so-called "equatorward diffuse emissions" in the equatorial plane.

Connerney et al. [1998] used the location of the Io footprint as observed by the IRTF IR telescope and by the HST to build the VIP4 magnetic field model. This model is made of a fourth-order multipole, plus an external contribution from an axisymmetric ring current. In order to constrain the weight of the different spherical harmonic coefficients, they used Pioneer 10 and 11 and Voyager 1 and 2 magnetic field measurements and tuned the coefficients such that the mapping of the Io orbit would fall as close as possible to the Io footpath (i.e., the locus of the Io footprint locations in a System III fixed reference frame). While this model prescribed that any point on the Io orbit would map to the Io footpath, the exact footprint location along this footpath was not constrained by observations. Based on a much larger number of highresolution HST images, Hess et al. [2011b] built a fifthorder multipole model, called VIPAL, constrained not only by the Io footpath, but also by the locations of the MAW spot along it.

The comparison of the shape of the footpaths of the different moons can also provide some useful information. Grodent et al. [2008] noticed that, in the northern hemisphere, the Io footpath on one side and the Europa and the Ganymede footpaths on the other side appear to diverge around $100^{\circ}$ in System III longitude. These authors suggested that this behavior could be related to a localized magnetic anomaly. They showed that they could fit well the three observed northern footpaths by adding a weak dipole located $\sim 17,000 \mathrm{~km}$ under the surface to a fourth-order spherical harmonics magnetic field model.

While the footprints can be used as constraints for modeling the internal magnetic field, they can also be useful for mapping features from the outer magnetosphere. Vogt et al. [2011] used the Ganymede footpath as a starting point to build a magnetic mapping model of Jupiter's auroral features to magnetospheric sources based on magnetic flux equivalence (see also Vogt and Kivelson [this volume]).

For a given System III longitude, the location of the Io footprint does not appear to change significantly from one epoch to another, but the same conclusion cannot be drawn for the Ganymede footprint. Grodent et al. [2008] analyzed two images acquired 5 years apart, and they noted a significant equatorward shift of $2^{\circ}$ for the Ganymede footprint and of $3^{\circ}$ for the main auroral emission, while the Io footprint location remains the same. They suggested that these motions stemmed from increased plasma sheet ring current, which caused an increased stretching of the magnetic field lines. The most probable cause for an increasing ring current is an enhancement of Io's volcanic plasma supply, which likely increased the density of the plasma sheet as well as the mass outflow rate.

\section{SIZE OF THE SATELLITE FOOTPRINTS}

On high-sensitivity HST FUV images, the spots usually appear roughly circular because the observation line of sight is generally perpendicular to the footpath and because the vertical scale height is similar to the length along the footpath. However, based on a subset of observations where the Io footprint was coaligned with the line of sight, Bonfond [2010] found that the Io footprint was a less than $200 \mathrm{~km}$ wide curtain, which corresponds to twice the size of Io at most. Based on another set of HST images on which the Io 
footprint is approximately perpendicular to the line of sight, Bonfond [2010] measured the full width at half maximum of the MAW spot and of the TEB spot to be $\sim 850 \mathrm{~km}$ long. This value corresponds to three to four times the projected size of Io along magnetic field lines. This indicates that the interaction region probably encompasses both Io and part of its wake, as suggested by Clarke et al. [2002]. It should, however, be noted that the $\sim 3000 \mathrm{~km}$ long spots discussed in the latter paper are the result of the juxtaposition of the three spots and not to a particularly extended unique one. On visible images acquired with Galileo's solid-state imaging (SSI) camera and with New Horizons' Long Range Reconnaissance Imager (LORRI) camera, the footprint looks like a $\sim 450 \mathrm{~km}$ wide circular patch [Vasavada et al., 1999] or like a $400 \mathrm{~km}$ wide and $1000 \mathrm{~km}$ high spot [Gladstone et al., 2007], respectively. Bonfond [2010] suggested that the smaller size of the visible spot could be caused by the limited sensitivity of the instruments. A similar effect has been observed when comparing the size of the Io footprint main spot obtained from the low-sensitivity faint object camera (FOC) aboard HST, also $400 \mathrm{~km}$ [Prangé et al., 1996], on one hand, and from the more sensitive STIS and ACS cameras aboard the same spacecraft on the other. As far as the Io footprint tail is concerned, its brightness progressively decreases downstream with an e-folding distance of $\sim 21,000$ $\mathrm{km}$ [Bonfond et al., 2009].

Some estimates of size of the other footprints' spots have also been published. It should, however, be noted that these studies did not discuss the discrimination between the vertical extent of the emissions and their horizontal width or the blurring caused by the motion of the footprint during the exposure with the same level of detail as those concerning the Io footprint. As far as Europa's footprint is concerned, Grodent et al. [2006] measured the spot's full width at half maximum to be $\sim 1100 \mathrm{~km}$ long. This distance is much longer than the projected diameter of the moon $(\sim 75 \mathrm{~km})$, indicating that the interaction region is probably much larger than the satellite itself. Moreover, these authors also observed a faint $\sim 7500 \mathrm{~km}$ long tail following the spot when Europa is close to the torus center. This result suggests that this auroral feature is the signature of an extended plasma plume downstream of Europa [Kivelson et al., 1999]. Grodent et al. [2009] estimated the surface of the Ganymede footprint to cover $\sim 5 \times 10^{5} \mathrm{~km}^{2}$. Mapped back in the equatorial plane, this surface would correspond to an 8 to 20 Ganymede radii wide region. They concluded that the interaction region is not restricted to Ganymede but also includes its mini-magnetosphere owing to its internal magnetic field. Finally, the Enceladus footprint observed by Pryor et al. [2011] also appears to map to a region extending up to 20 Enceladus radii $\left(R_{E}\right)$ downstream of the moon. Depending whether the extent of the spot corresponded to the vertical extent of the spot or to its latitudinal width, the authors noted that the radial extent of the interaction region would range from 0 to $20 R_{E}$. The large footprint extent also suggests that the interaction region covers the extended neutral plume exhausted by the geysers rather than the satellite alone.

\section{VERTICAL EXTENT OF THE IO FOOTPRINT}

The isolation of the Io footprint from the other auroral emissions at Jupiter allows direct observations of its vertical extent above the planetary limb (associated with the 1 bar level). The more energetic precipitating electrons are, the more deeply into the atmosphere they penetrate, and the lower the auroral emissions will be. As far as the tail emissions are concerned, the altitude of the brightness peak is $\sim 900 \mathrm{~km}$ above limb, and it remains constant whatever the distance from the spots, indicating that the energy of the precipitating electrons does not change with the distance from the spots. The scale height of the tail emissions is on the order of $\sim 430 \mathrm{~km}$. The peak altitude and the scale height of the MAW spots are approximately similar [Bonfond, 2010]. Bonfond et al. [2009] concluded that the electron distribution that best matches the data was a kappa distribution with a mean energy of $1 \mathrm{keV}$ and a spectral index of 2.3. This estimate of the mean energy contrasts with the $55 \mathrm{keV}$ deduced from measurements of the attenuation of FUV emissions below $135 \mathrm{~nm}$ following methane absorption [Gérard et al., 2002]. This discrepancy most probably arises from our poor knowledge of the Jovian auroral atmosphere composition profile. Hess et al. [2010] modeled the energy distribution of electrons accelerated by inertial Alfvén waves, and resulting power law energy distributions are consistent with the broadness of the MAW spot vertical profile. Delamere et al. [2003] proposed that, contrary to the spots, the tail emissions are generated by a steady state electrodynamic process related to the acceleration of the initially stagnant plasma at Io back to corotation with the planet. In this steady state framework, Ergun et al. [2009] concluded that the tail emissions were generated by electrons accelerated by a quasistatic electric field on the order of $1 \mathrm{kV}$, in accordance with the mean energy deduced from the tail altitude. Nevertheless, Bonfond et al. [2009] argued that such a quasi-static electric field would lead to quasi-monoenergetic electrons, which would then create a narrower vertical extent than observed. While the altitudes of the main spot and of the tail are similar, the TEB spot vertical profile appears to peak $200 \mathrm{~km}$ lower in the atmosphere. As a consequence, the impinging electron energy is thus expected to be approximately four times higher than for the other two features. This result confirms that the different spots have different origins, and the model of Hess 
et al. [2010] indeed predicted different energy distributions for the electrons directly precipitating into the atmosphere and those accelerated toward the opposite hemisphere.

\section{BRIGHTNESS OF THE SATELLITE FOOTPRINTS}

Using increasingly large image data sets, recent studies of the Io footprint main spot brightness indicated that the brightness of the main spot appeared to peak when Io is close to the torus center [Gérard et al., 2006; Serio and Clarke, 2008; Wannawichian et al., 2010]. Wannawichian et al. [2010] estimated that the brightness peaked at $107^{\circ}$ and at $287^{\circ} \pm 8^{\circ}$ because the denser plasma would generate a stronger interaction. When Io approached the torus northern or southern boundaries, the brightness appeared to be down to $\sim 10$ times lower. An alternative reason for the enhanced brightening of the main spot when Io is close to the torus center is the fact that it is merged with the TEB spot at that time.

Bonfond et al. [2007] observed variations of the Io footprint on time scales of a minute and found brightness variations up to 50\%. Moreover, Hess et al. [2009] identified fluctuations with a similar time scale when analyzing Iorelated S-burst radio emissions. Indeed, their results suggest the presence of vertically drifting double layer structures with a regeneration time of $200 \mathrm{~s}$. The electric field of these double layers does not seem to be the main cause for the electron precipitation [Hess and Delamere, this volume]. However, a possible scenario is that these variable acceleration structures could provide the electrons with some additional energy and thus trigger the fast brightness variations.

Wannawichian et al. [2010] also studied the Europa footprint brightness, but they did not find evidence for variations of the footprint brightness with the centrifugal latitude.

As far as the Ganymede footprint is concerned, Grodent et al. [2009] identified three different time scales for brightness variations. As in the Io case, the longest time scale $(\sim 10 \mathrm{~h})$ is related to the location of Ganymede in the plasma sheet, the footprint being approximately twice brighter when Ganymede approaches the center. The second time scale ranges between 10 and $40 \mathrm{~min}$ and has been tentatively associated with interactions between the Ganymede mini-magnetosphere and localized magnetospheric inhomogeneities, such as plasma injections. The shortest time scale is on the order of 1-2 min, and two scenarii have been proposed. Either these variations are triggered by bursty reconnections at the Ganymede magnetopause [Jia et al., 2010], or they are related to double layer regeneration as suggested for the Io footprint.

The Enceladus footprint is the most elusive of all footprints. It has only been detected in a few percent of the UVIS observations, which implies that its brightness only occasionally reaches the instrument detection threshold [Pryor et al., 2011]. Even when it is detected, its brightness appears to vary by a factor of 3 within $5 \mathrm{~h}$. This behavior was attributed to the variability of Enceladus's cryovolcanism.

Another quantity to extract from footprint images is the integrated emitted power, which is directly related to the total precipitated power. Hess et al. [2010] compared this quantity to the available power in the interaction region for Io, and Hess et al. [2011a] extended this study to Europa and Enceladus. Computing the amount of energy escaping the torus and the efficiency of the power transmission to the precipitating particles, they concluded that the large-scale Alfvén waves need to filament into smaller structures to be able to generate the observed auroral brightness.

\section{CONCLUSIONS}

The strength of their magnetic field, their rapid rotation, and the presence of generous internal plasma sources make the Jovian and Kronian magnetospheres very different from the Earth's magnetosphere. One of the consequences of these three elements is the occurrence of localized aurora close to the feet of the field lines passing through the satellites Io, Europa, Ganymede at Jupiter, and Enceladus at Saturn.

The direct relationship between the satellites and the auroral footprints provide unique landmarks in the planetary magnetosphere, which have been used to improve the Jovian magnetic field models and investigate the variability of the ring current.

A major recent advance in the field is the finding that the Alfvén waves generated by the strong moon-magnetosphere interactions accelerate electrons in both directions along the field lines. This mechanism simultaneously explains the multiplicity of the Io footprint spots and the detection of electron beams affecting the ionization processes near the satellite. It also accounts for the vertical extent of the different features of the Io footprint.

Whatever the satellite under consideration is, the size of its footprint spots appears to map to a region much wider than the moon. This is a clear indication that the satellite-magnetosphere interactions are not restricted to the satellites themselves, but more likely include either parts of the neutral cloud that surrounds and follows them in the case of Io, Europa, and Enceladus or its mini-magnetosphere for Ganymede. It is, however, surprising that Io appears to be the satellite with the smallest interaction region, and further analyses of the other footprints' sizes should be carried out to reduce measurement uncertainties.

Finally, studies of the Io and Ganymede footprint brightness indicate that the position of the satellites in the Jovian 
magnetic field does strongly control the footprint brightness. However, other variation time scales have been identified, suggesting that many other processes are simultaneously at play. The huge emitted power of these footprints, up to a few GW for the Io footprint, a few $100 \mathrm{MW}$ for the Europa footprint, and a few MW for the Enceladus footprint [Hess et al., 2011a], suggests filamentation of the Alfvén waves as they travel from the satellites to the planetary ionospheres.

Acknowledgments. The author thanks J.-C. Gérard and D. Grodent for their help and constructive comments during the preparation of this manuscript. This study was supported by the PRODEX program managed by ESA in collaboration with the Belgian Federal Science Policy Office.

\section{REFERENCES}

Bigg, E. K. (1964), Influence of the satellite Io on Jupiter's decametric emission, Nature, 203, 1008-1010.

Bonfond, B. (2010), The 3-D extent of the Io UV footprint on Jupiter, J. Geophys. Res., 115, A09217, doi:10.1029/2010JA 015475.

Bonfond, B., J.-C. Gérard, D. Grodent, and J. Saur (2007), Ultraviolet Io footprint short timescale dynamics, Geophys. Res. Lett., 34, L06201, doi:10.1029/2006GL028765.

Bonfond, B., D. Grodent, J.-C. Gérard, A. Radioti, J. Saur, and S. Jacobsen (2008), UV Io footprint leading spot: A key feature for understanding the UV Io footprint multiplicity?, Geophys. Res. Lett., 35, L05107, doi:10.1029/2007GL032418.

Bonfond, B., D. Grodent, J.-C. Gérard, A. Radioti, V. Dols, P. A. Delamere, and J. T. Clarke (2009), The Io UV footprint: Location, inter-spot distances and tail vertical extent, J. Geophys. Res., 114, A07224, doi:10.1029/2009JA014312.

Clarke, J. T., et al. (1996), Far-ultraviolet imaging of Jupiter's aurora and the Io "footprint," Science, 274, 404-409.

Clarke, J. T., et al. (2002), Ultraviolet emissions from the magnetic footprints of Io, Ganymede and Europa on Jupiter, Nature, 415, 997-1000.

Connerney, J. E. P., and T. Satoh (2000), The $\mathrm{H}_{3}^{+}$ion: A remote diagnostic of the Jovian magnetosphere, Philos. Trans. R. Soc. London, Ser. A, 358(1774), 2471-2483.

Connerney, J. E. P., R. Baron, T. Satoh, and T. Owen (1993), Images of excited $\mathrm{H}_{3}^{+}$at the foot of the Io flux tube in Jupiter's atmosphere, Science, 262, 1035-1038.

Connerney, J. E. P., M. H. Acuña, N. F. Ness, and T. Satoh (1998), New models of Jupiter's magnetic field constrained by the Io flux tube footprint, J. Geophys. Res., 103(A6), 11,929-11,939.

Delamere, P. A., F. Bagenal, R. Ergun, and Y.-J. Su (2003), Momentum transfer between the Io plasma wake and Jupiter's ionosphere, J. Geophys. Res., 108(A6), 1241, doi:10.1029/2002JA009530.

Dols, V., P. A. Delamere, and F. Bagenal (2008), A multispecies chemistry model of Io's local interaction with the Plasma Torus, J. Geophys. Res., 113, A09208, doi:10.1029/2007JA012805.
Ergun, R. E., L. Ray, P. A. Delamere, F. Bagenal, V. Dols, and Y.-J. $\mathrm{Su}$ (2009), Generation of parallel electric fields in the Jupiter-Io torus wake region, J. Geophys. Res., 114, A05201, doi:10.1029/ 2008JA013968.

Gérard, J.-C., J. Gustin, D. Grodent, P. Delamere, and J. T. Clarke (2002), Excitation of the FUV Io tail on Jupiter: Characterization of the electron precipitation, J. Geophys. Res., 107(A11), 1394, doi:10.1029/2002JA009410.

Gérard, J.-C., A. Saglam, D. Grodent, and J. T. Clarke (2006), Morphology of the ultraviolet Io footprint emission and its control by Io's location, J. Geophys. Res., 111, A04202, doi:10.1029/ 2005JA011327.

Gladstone, G. R., et al. (2007), Jupiter's nightside airglow and aurora, Science, 318, 229-231, doi:10.1126/science.1147613.

Gledhill, J. A. (1967), Magnetosphere of Jupiter, Nature, 214, 155156, doi:10.1038/214155a0.

Goertz, C. K. (1980), Io's interaction with the plasma torus, J. Geophys. Res., 85(A6), 2949-2956.

Goldreich, P., and D. Lynden-Bell (1969), Io, a Jovian unipolar inductor, Astrophys. J., 156, 59-78.

Grodent, D., J.-C. Gérard, J. Gustin, B. H. Mauk, J. E. P. Connerney, and J. T. Clarke (2006), Europa's FUV auroral tail on Jupiter, Geophys. Res. Lett., 33, L06201, doi:10.1029/2005GL025487.

Grodent, D., B. Bonfond, J.-C. Gérard, A. Radioti, J. Gustin, J. T. Clarke, J. Nichols, and J. E. P. Connerney (2008), Auroral evidence of a localized magnetic anomaly in Jupiter's northern hemisphere, J. Geophys. Res., 113, A09201, doi:10.1029/2008JA013185.

Grodent, D., B. Bonfond, A. Radioti, J.-C. Gérard, X. Jia, J. D. Nichols, and J. T. Clarke (2009), Auroral footprint of Ganymede, J. Geophys. Res., 114, A07212, doi:10.1029/2009JA014289.

Gurnett, D. A., and C. K. Goertz (1981), Multiple Alfven wave reflections excited by Io: Origin of the Jovian decametric arcs, J. Geophys. Res., 86(A2), 717-722.

Gurnett, D. A., and W. R. Pryor (2012), Auroral processes associated with Saturn's moon Enceladus, in Auroral Phenomenology and Magnetospheric Processes: Earth and Other Planets, Geophys. Monogr. Ser., doi:10.1029/2011GM001174, this volume.

Hess, S., and P. A. Delamere (2012), Satellite-induced electron acceleration and related auroras, in Auroral Phenomenology and Magnetospheric Processes: Earth and Other Planets, Geophys. Monogr. Ser., doi:10.1029/2011GM001175, this volume.

Hess, S., P. Zarka, F. Mottez, and V. B. Ryabov (2009), Electric potential jumps in the Io-Jupiter flux tube, Planet. Space Sci., 57, 23-33, doi:10.1016/j.pss.2008.10.006.

Hess, S. L. G., P. Delamere, V. Dols, B. Bonfond, and D. Swift (2010), Power transmission and particle acceleration along the Io flux tube, J. Geophys. Res., 115, A06205, doi:10.1029/2009JA 014928.

Hess, S. L. G., P. A. Delamere, V. Dols, and L. C. Ray (2011a), Comparative study of the power transferred from satellitemagnetosphere interactions to auroral emissions, J. Geophys. Res., 116, A01202, doi:10.1029/2010JA015807.

Hess, S. L. G., B. Bonfond, P. Zarka, and D. Grodent (2011b), Model of the Jovian magnetic field topology constrained by the 
Io auroral emissions, J. Geophys. Res., 116, A05217, doi:10. 1029/2010JA016262.

Hill, T. W., and V. M. Vasyliunas (2002), Jovian auroral signature of Io's corotational wake, J. Geophys. Res., 107(A12), 1464, doi:10. 1029/2002JA009514.

Jacobsen, S., F. M. Neubauer, J. Saur, and N. Schilling (2007), Io's nonlinear MHD-wave field in the heterogeneous Jovian magnetosphere, Geophys. Res. Lett., 34, L10202, doi:10.1029/2006GL 029187.

Jacobsen, S., J. Saur, F. M. Neubauer, B. Bonfond, J.-C. Gérard, and D. Grodent (2010), Location and spatial shape of electron beams in Io's wake, J. Geophys. Res., 115, A04205, doi:10.1029/ 2009JA014753.

Jia, X., M. G. Kivelson, K. K. Khurana, and R. J. Walker (2009), Magnetic fields of the satellites of Jupiter and Saturn, Space Sci. Rev., 152, 271-305, doi:10.1007/s11214-009-9507-8.

Jia, X., R. J. Walker, M. G. Kivelson, K. K. Khurana, and J. A. Linker (2010), Dynamics of Ganymede's magnetopause: Intermittent reconnection under steady external conditions, J. Geophys. Res., 115, A12202, doi:10.1029/2010JA015771.

Kivelson, M. G., K. K. Khurana, D. J. Stevenson, L. Bennett, S. Joy, C. T. Russell, R. J. Walker, C. Zimmer, and C. Polanskey (1999), Europa and Callisto: Induced or intrinsic fields in a periodically varying plasma environment, J. Geophys. Res., 104(A3), 4609-4625.

Kivelson, M. G., F. Bagenal, W. S. Kurth, F. M. Neubauer, C. Paranicas, and J. Saur (2004), Magnetospheric interactions with satellites, in Jupiter: The Planet, Satellites and Magnetosphere, edited by F. Bagenal, T. E. Dowling, and W. B. McKinnon, pp. 513-536, Cambridge Univ. Press, Cambridge, U. K.

Neubauer, F. M. (1980), Nonlinear standing Alfvén wave current system at Io: Theory, J. Geophys. Res., 85(A3), 1171-1178.

Pontius, D. H., Jr., and T. W. Hill (2006), Enceladus: A significant plasma source for Saturn's magnetosphere, J. Geophys. Res., 111, A09214, doi:10.1029/2006JA011674.

Prangé, R., D. Rego, D. Southwood, P. Zarka, S. Miller, and W. Ip (1996), Rapid energy dissipation and variability of the Io-Jupiter electrodynamic circuit, Nature, 379, 323-325, doi:10.1038/ 379323a0.

Pryor, W. R., et al. (2011), The auroral footprint of Enceladus on Saturn, Nature, 472, 331-333, doi:10.1038/nature09928.
Radioti, A., A. T. Tomás, D. Grodent, J.-C. Gérard, J. Gustin, B. Bonfond, N. Krupp, J. Woch, and J. D. Menietti (2009), Equatorward diffuse auroral emissions at Jupiter: Simultaneous HST and Galileo observations, Geophys. Res. Lett., 36, L07101, doi:10.1029/2009GL037857.

Saur, J., F. M. Neubauer, D. F. Strobel, and M. E. Summers (2002), Interpretation of Galileo's Io plasma and field observations: I0, I24, and I27 flybys and close polar passes, J. Geophys. Res., 107(A12), 1422, doi:10.1029/2001JA005067.

Saur, J., F. M. Neubauer, J. E. P. Connerney, P. Zarka, and M. G. Kivelson (2004), Plasma interaction of Io with its plasma torus, in Jupiter: The Planet, Satellites and Magnetosphere, edited by F. Bagenal, T. E. Dowling, and W. B. McKinnon, pp. 537-560, Cambridge Univ. Press, Cambridge, U. K.

Serio, A. W., and J. T. Clarke (2008), The variation of Io's auroral footprint brightness with the location of Io in the plasma torus, Icarus, 197, 368-374, doi:10.1016/j.icarus.2008.03.026.

Vasavada, A. R., A. H. Bouchez, A. P. Ingersoll, B. Little, C. D. Anger, and Galileo SSI Team (1999), Jupiter's visible aurora and Io footprint, J. Geophys. Res., 104(E11), 27,133-27,142.

Vogt, M. F., and M. G. Kivelson (2012), Relating Jupiter's auroral features to magnetospheric sources, in Auroral Phenomenology and Magnetospheric Processes: Earth and Other Planets, Geophys. Monogr. Ser., doi:10.1029/2011GM001181, this volume.

Vogt, M. F., M. G. Kivelson, K. K. Khurana, R. J. Walker, B. Bonfond, D. Grodent, and A. Radioti (2011), Improved mapping of Jupiter's auroral features to magnetospheric sources, J. Geophys. Res., 116, A03220, doi:10.1029/2010JA016148.

Wannawichian, S., J. T. Clarke, and J. D. Nichols (2010), Ten years of Hubble Space Telescope observations of the variation of the Jovian satellites' auroral footprint brightness, J. Geophys. Res., 115, A02206, doi:10.1029/2009JA014456.

Wilkinson, M. H. (1998), Evidence for periodic modulation of Jupiter's decametric radio emission, J. Geophys. Res., 103(E9), 19,985-19,991.

Williams, D. J., and R. M. Thorne (2003), Energetic particles over Io's polar caps, J. Geophys. Res., 108(A11), 1397, doi:10.1029/ 2003JA009980.

B. Bonfond, Laboratoire de Physique Atmosphérique et Planétaire, Université de Liège, B-4000 Liège, Belgium. (b.bonfond@ulg. ac.be) 